\title{
Repercussões da Exposição à Violência Conjugal nas Características Emocionais dos Filhos: Revisão Sistemática da Literatura
}

\author{
Naiana Dapieve Patias ${ }^{1}$ \\ Tatiele Jacques Bossi \\ Programa de Pós-Graduação em Psicologia, Universidade Federal do Rio Grande do Sul, \\ Porto Alegre, Rio Grande do Sul, Brasil \\ Débora Dalbosco Dell'Aglio \\ Departamento de Psicologia do Desenvolvimento e da Personalidade da Universidade \\ Federal do Rio Grande do Sul, Porto Alegre, Rio Grande do Sul, Brasil
}

\section{Resumo}

Este estudo teve como objetivo revisar a produção científica sobre as repercussões da exposição direta e indireta à violência conjugal nas características emocionais dos filhos, com idades de zero a 12 anos. Foram selecionados 14 artigos empíricos publicados nos últimos 12 anos (2002-2014), sendo um nacional e 13 internacionais indexados em diferentes bases de dados, a partir da combinação de descritores sobre o tema investigado. Os resultados sugerem uma diversidade de repercussões da exposição à violência no desenvolvimento dos filhos a curto, médio e longo prazo, principalmente relativas a problemas de comportamentos internalizantes e externalizantes acessados, na sua maioria, através do relato da mãe e/ou da própria criança. Quanto aos aspectos metodológicos dos artigos incluídos na seleção final, todos eles são quantitativos e a maioria transversal. Discute-se sobre a necessidade de novas investigações sobre o tema, principalmente no Brasil. As limitações dos estudos investigados são destacadas, sugerindo-se novos trabalhos sobre essa temática, que possam dar mais visibilidade ao fenômeno.

Palavras-chave: Violência conjugal, infância, características emocionais, revisão sistemática da literatura.

\section{Repercussions of the Exposure to Marital Violence on Emotional Characteristics of Children: A Systematic Review of the Literature}

\begin{abstract}
This study aimed to review scientific production about the effects of direct and indirect exposure to marital violence on emotional characteristics of children aged 0-12 years (2002-2014). Based on a combination of descriptors on the subject, 14 empirical papers published in the last 12 years, one national and 13 international indexed in different databases were selected. The results suggest a variety of effects related to the exposure to violence on development of children in short, medium and long term, especially concerning externalizing and internalizing behavior problems that were mostly accessed by the report of the mother and /or the child himself. Regarding methodological aspects of the articles included in the final selection, all of them are quantitative and most of them are cross-sectional. The need for additional research on this theme, mainly in Brazil, is also discussed. The limitations of the investigated
\end{abstract}

1 Endereço para correspondência: Rua Ramiro Barcelos, 2600, sala 115, Bairro Santa Cecília, Porto Alegre, RS, 90035-003. E-mail: naipatias@hotmail.com 
studies are pointed out and new researches on this subject are suggested in order to give more visibility to this phenomenon.

Keywords: Marital violence, childhood, emotional characteristics, sistematic review.

\section{Repercusiones de la Exposición a la Violencia Doméstica en las Características Emocionales de los Niños: Revisión Sistemática de la Literatura}

\section{Resumen}

Este estudio tuvo como objetivo revisar la literatura científica sobre los efectos de la exposición directa e indirecta a la violencia doméstica sobre las características emocionales de los niños de 0 a 12 años de edad. Fueron seleccionados 14 artículos empíricos publicados en los últimos 12 años (2002-2014), uno nacional y 13 internacionales indexados en diferentes bases de datos, a partir de la combinación de descriptores sobre el tema investigado. Los resultados sugieren una diversidad de efectos de la exposición a la violencia en el desarrollo de los niños en el corto, mediano y largo plazo, principalmente relacionados con los problemas de internalización y externalización de conductas a los cuales se tuvo acceso principalmente a través del informe de la madre y / o del propio niño. En cuanto a los aspectos metodológicos de los artículos incluidos en la selección final, todos son cuantitativos y la mayoría es transversal. Se discute acerca de la necesidad de más investigación sobre el tema, especialmente en Brasil. Las limitaciones de los estudios investigados son resaltadas, sugiriéndose nuevos trabajos en este tema que puedan dar más visibilidad a este fenómeno.

Palabras clave: Violencia conyugal, la infancia, las características emocionales, revisión de la literatura.

A violência tem sido considerada um problema de saúde pública que pode gerar consequências inestimáveis à saúde como um todo, podendo, inclusive levar a morte (Minayo, 2006). Mais especificamente, a violência que ocorre no contexto da família é entendida como um problema social que afeta a todas as camadas socioeconômicas, interferindo no sistema familiar como um todo e atingindo, principalmente, mulheres, crianças e adolescentes (Araújo, 2002). Dessa forma, tornou-se uma das principais preocupações dos profissionais da área da saúde, pelo fato de não ser esperado que a família - contexto de proteção e primeiro ambiente de desenvolvimento humano - seja um ambiente violento (Abranches \& Assis, 2011; Araújo, 2002; Ministério da Saúde, 2001).

Considerada como toda ação ou omissão que prejudique o bem-estar, integridade física e psicológica, bem como o desenvolvimento de membros da família, a violência intrafamiliar é cometida por um membro ou pessoa que assu- me funções parentais, dirigida a outro membro, mesmo sem laços de consanguinidade (World Health Organization, 2002). Essa violência pode ocorrer no espaço doméstico ou fora desse, e diz respeito às relações entre vítimas e perpetradores da violência, mais do que ao espaço físico onde ocorre. Esse conceito diferencia-se da violência doméstica, pois essa se refere ao espaço físico privado, mais do que às relações de afeto, podendo acontecer entre pessoas que ali convivem, como empregados, por exemplo (Ministério da Saúde, 2001).

Dentro do contexto da violência intrafamiliar, pode ocorrer a violência de pais contra seus filhos, de forma direta, através da violência física, psicológica, sexual ou negligência. Neste mesmo contexto, a violência conjugal, considerada uma forma de violência indireta aos filhos (Benetti, 2006; Ministério da Saúde, 2001), também pode estar presente. Segundo Colossi e Falcke (2013), a violência conjugal pode ser compreendida a partir de duas concepções teóri- 
cas que, embora complementares possuem focos diferentes: (a) perspectiva feminista; e (b) perspectiva sistêmica. A primeira considera a violência conjugal a partir da diferença de gênero e subversão feminina ao masculino, ainda presente na sociedade atual. Dessa forma, a violência conjugal é um fenômeno unidirecional - homem (agressor) e mulher (vítima), de forma que o atendimento e a proteção devem ser realizados com a mulher e a punição com o homem. Por outro lado, não excludente, mas complementar à perspectiva feminista, a segunda abordagem, sistêmica, considera a violência conjugal como um fenômeno da conjugalidade - da relação do casal. Assim, a mulher também está implicada na relação violenta de forma a fazer parte de sua manutenção. Essa abordagem não tenta culpabilizar a mulher, mas compreende a violência como um fenômeno circular e como um modelo de relação do casal. Essa percepção influencia a forma como são orientados os casos que, geralmente, podem ser submetidos à terapia de casal (Colossi \& Falcke, 2013).

Em uma perspectiva feminista, considera-se que a violência pode ocorrer da mulher para o homem, mas sendo mais comum ocorrer do homem contra a mulher (Cortez, Cruz, \& Souza, 2013; Oliveira \& Gomes, 2011; K. Santos et al., 2014). Nessa abordagem, a violência conjugal é considerada uma das principais causas de morte de mulheres no Brasil, ocupando a sétima posição no ranking internacional de homicídios femininos pela violência sofrida (Waiselfisz, 2012). De fato, dados de estudos brasileiros (Bhona, Lourenço, \& Brum, 2011; Brum, Lourenço, Gebara, \& Ronzani, 2013; Waiselfisz, 2012) estimam que, no Brasil, uma a cada cinco mulheres sofrem de alguma forma de violência durante a vida, tendo como principais agressores maridos ou companheiros. Outros estudos, também nacionais, estimam uma prevalência maior de violência - uma para cada três mulheres (Miranda, Paula, \& Bordin, 2010; Vieira, Perdona, $\&$ Santos, 2011). Segundo o mapa de homicídios de mulheres no Brasil, de 1980 a 2010 a taxa de mortes aumentou em 217,6\%, havendo um decréscimo em 2007, no primeiro ano de vigência da Lei Maria da Penha (Waiselfisz, 2012). Essa
Lei, 11.340 de 7 de agosto de 2006 criou mecanismos para coibir a violência doméstica contra mulheres estabelecendo medidas de proteção e assistência às vítimas.

Pela perspectiva sistêmica, quando a violência conjugal ocorre, toda a família sofre as consequências da violência, mesmo que de modo indireto. A respeito dessa vitimização indireta dos filhos, através do testemunho da violência conjugal sofrida, geralmente, pela mulher, estudos brasileiros são realizados, mas em menor número se comparados com estudos que enfatizam a exposição direta de crianças e adolescentes à violência (Araújo, 2002; Braga \& Dell'Aglio, 2012; Marasca, Colossi, \& Falcke, 2013). No entanto, presenciar ou testemunhar violência, principalmente no contexto da família, tem sido preocupação constante de pesquisadores internacionais, já que não apenas a exposição direta à violência como vítima, mas também a exposição indireta, como testemunha da violência, resulta em consequências ao desenvolvimento humano (Almeida, Miranda, \& Lourenço, 2013; Hardaway, McLoyd, \& Wood, 2012; Ho \& Cheung, 2010; Mrug \& Windle, 2010), como por exemplo: problemas de comportamento internalizante (depressão e ansiedade; Hanson et al., 2008; Hardaway et al., 2012; Margolin, Vickerman, Oliver, \& Gordis, 2010; Mrug \& Windle, 2010), problemas de comportamento externalizante (agressão e comportamento antissocial; Hardaway et al., 2012; Ho \& Cheung, 2010; Williams \& Stelko-Pereira, 2008), Transtorno de Estresse Pós-Traumático (Benetti, Pizetta, Schwartz, Hass, \& Melo, 2010; Williams, D’Affonseca, Correia, \& Albuquerque, 2011), suicídio (Haynie, Petts, Maimon, \& Piquero, 2009) e sintomas somáticos (Hart, Hodgkinson, Belcher, Hyman, \& Cooley-Strickland, 2012). Resultados negativos em termos educacionais, também são encontrados: evasão escolar (Haynie et al., 2009), problemas de comportamento na escola e menor desempenho escolar (Brancalhone, Fogo, \& Williams, 2004).

Devido às preocupações em relação à vitimização direta e indireta à violência, por parte das crianças, este artigo teve como objetivo revisar a produção científica nacional e internacional 
sobre as repercussões da exposição à violência conjugal nas características emocionais dos filhos, com idades de zero a 12 anos. Foram consideradas as repercussões da exposição à violência na infância, tendo em vista a importância desse período, já que diversos autores, principalmente de base psicanalítica (Bowlby, 1989; Mahler, Pine, \& Bergman, 1975/1977; Winnicott, 1945/2000), consideram as experiências iniciais e aquelas que ocorrem na infância como as mais importantes no desenvolvimento emocional e saúde mental.

\section{Método}

A busca dos artigos foi realizada através das bases de dados eletrônicas: (a) SciELO (Scientific Eletronic Library Online); (b) PePSIC (Periódicos Eletrônicos em Psicologia); (c) MEDLINE (Pubmed); e, (d) PsycINFO. Para a busca de artigos, foram utilizados os seguintes descritores combinados com operadores booleanos: (psychol*effect OR effect OR impact OR consequence) AND (domestic violence OR intra-

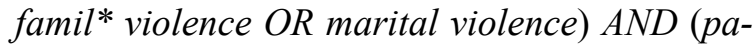
rents OR child* OR adolescen*) ${ }^{2}$, selecionando como período de busca os anos de 2002 a 2014 (até maio de 2014).

Ao todo, foram encontrados 736 documentos, distribuídos entre as bases de dados, da seguinte forma: 20 na base SciELO, nenhum na PePSIC, 458 na Medline (PubMed) e 258 na PsycINFO. Os artigos encontrados foram classificados a partir dos seguintes critérios de inclusão: (a) artigo empírico nacional e internacional escrito em inglês, português ou espanhol; (b) ter como participantes, ou como foco do estudo crianças de zero a 12 anos; (c) estudos cujo foco principal foram as repercussões da exposição à violência conjugal nas características emocionais dos filhos. Foram excluídas aquelas referências repetidas entre as bases de dados

2 Quando o asterisco (*) foi incluído no radical da palavra significa que, na busca, foram consideradas palavras com o mesmo radical. Por exemplo, em adolescen* foram incluídos adolescence e adolescent, ampliando a busca. (quatro artigos), escritas em outros idiomas que não os destacados nos critérios de inclusão (12 artigos), revisões de literatura (87 artigos), teses, dissertações e outros tipos de trabalhos que não eram artigos empíricos (65 documentos). Além disso, foram excluídos os artigos que não tinham como participantes ou foco da pesquisa crianças de zero a 12 anos (404 artigos), que não apresentavam como foco de estudo a violência conjugal (124 artigos) e que não abordavam as repercussões da exposição à violência conjugal nas características emocionais dos filhos (18 artigos). A partir dessa primeira seleção, foram considerados 22 artigos, que foram lidos na íntegra por duas das autoras. Após a leitura, outros artigos foram retirados da amostra por apresentarem simultaneamente participantes crianças e adolescentes, sem diferenciar resultados por idade (oito artigos). Ao final, 14 artigos foram considerados para o presente estudo, sendo lidos e classificados por consenso por duas das autoras. A Figura 1 apresenta o fluxo de seleção dos artigos, a partir dos critérios de inclusão e exclusão. As informações dos estudos selecionados são apresentadas na Tabela 1 .

\section{Resultados}

Os artigos foram classificados em quatro grandes categorias temáticas, definidas a priori, a saber: (a) Caracterização das amostras; (b) Características metodológicas; (c) Características emocionais infantis; e, (d) Repercussões da exposição à violência conjugal nas características emocionais infantis. A seguir serão destacadas cada uma das categorias e ilustradas a partir dos artigos considerados neste estudo.

\section{Caracterização das Amostras}

Nesta categoria foram descritas as características dos participantes dos estudos. Observou-se que na maior parte dos artigos ( $\mathrm{A} 1^{3}, \mathrm{~A} 3, \mathrm{~A} 5$, A6, A9, A10, A11, A12, A13, A14) a amostra

3 Os artigos estão elencados em ordem numérica segundo Tabela 1 e serão apresentados dessa forma no decorrer do artigo. 


\begin{tabular}{|l|l|}
$\begin{array}{l}\text { Total de artigos gerados pela busca: } \\
(n=\mathbf{7 3 6})\end{array}$ \\
MEDLINE $(n=458)$ \\
$\begin{array}{ll}\text { PsycINFO }(n=258) \\
\text { SciELO }(n=20)\end{array}$ & Artigos excluídos \\
PePSIC $(n=0)$ & Motivos: \\
& - Artigos repetidos $(n=4)$ \\
& - Artigos em outro idioma $(n=12)$ \\
& - Artigos de revisão $(n=87)$ \\
& -Teses, dissertações e outros documentos $(n=65)$ \\
& -Participantes adolescentes ou adultos $(n=404)$ \\
& -Foco não era violência conjugal $(n=124)$ \\
& -Não abordava repercussões da violência $(n=18)$ \\
\hline
\end{tabular}

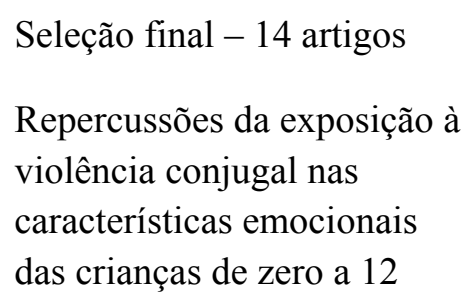

Artigos excluídos

Motivos:

- Artigos repetidos $(n=4)$

Artigos em outro idioma $(n=12)$

Artigos de revisão $(n=87)$

-Foco não era violência conjugal $(n=124)$

abordava repercussões da violência $(n=18)$

Figura 1. Diagrama do fluxo de seleção dos artigos.

Tabela 1

Informações dos Artigos Selecionados em Termos de Título, Autores, Ano de Publicação e Objetivos do Estudo ( $\mathrm{N}=14)$

\begin{tabular}{|c|c|c|c|}
\hline Título do artigo & Autores & $\begin{array}{c}\text { Ano de } \\
\text { publicação }\end{array}$ & Objetivo \\
\hline $\begin{array}{l}\text { A1 - The impact of domestic } \\
\text { violence on the maternal-child } \\
\text { relationship and preschool-age } \\
\text { children's functioning }\end{array}$ & $\begin{array}{l}\text { Levendosky, } \\
\text { Huth-Bocks, } \\
\text { Shapiro, } \\
\text { \& Semel }\end{array}$ & 2003 & $\begin{array}{c}\text { Examinar o papel da relação mãe-criança } \\
\text { como um mediador dos efeitos da } \\
\text { violência nas crianças }\end{array}$ \\
\hline $\begin{array}{l}\text { A } 2 \text { - Marital violence, co- } \\
\text { parenting, and family-level } \\
\text { processes in relation to children's } \\
\text { adjustment }\end{array}$ & Katz \& Low & 2004 & $\begin{array}{l}\text { Compreender a relação entre violência } \\
\text { doméstica, coparentalidade e processos } \\
\text { familiares e sua relação com o ajustamento } \\
\text { psicológico infantil }\end{array}$ \\
\hline $\begin{array}{l}\text { A3- Siblings in domestically } \\
\text { violent families: Experiences } \\
\text { of interparent conflict and } \\
\text { adjustment problems }\end{array}$ & $\begin{array}{l}\text { Skopp, Manke, } \\
\text { McDonald, } \\
\text { \& Jouriles }\end{array}$ & 2005 & $\begin{array}{c}\text { Examinar se irmãos expostos à violência } \\
\text { conjugal diferem na experiência desse } \\
\text { conflito, e se as diferenças estão associadas } \\
\text { ao ajustamento emocional }\end{array}$ \\
\hline $\begin{array}{l}\text { A4 - Domestic violence, emotion } \\
\text { coaching, and child adjustment }\end{array}$ & $\begin{array}{c}\text { Katz \& } \\
\text { Windecker-Nelson }\end{array}$ & 2006 & $\begin{array}{l}\text { Examinar opiniões e sentimentos dos pais } \\
\text { em contexto de violência doméstica quanto } \\
\text { a conversas com os filhos }\end{array}$ \\
\hline $\begin{array}{l}\text { A5- The mediating role of } \\
\text { parenting stress in the relation } \\
\text { between intimate partner violence } \\
\text { and child adjustment }\end{array}$ & $\begin{array}{l}\text { Owen, Kaslow, } \\
\text { \& Thompson }\end{array}$ & 2006 & $\begin{array}{l}\text { Examinar o papel do estresse parental } \\
\text { materno na relação entre violência } \\
\text { conjugal e problemas emocionais e } \\
\text { comportamentais nos filhos }\end{array}$ \\
\hline $\begin{array}{l}\text { A6- Variations in parenting stress } \\
\text { in African-American battered } \\
\text { women: Implications for children's } \\
\text { adjustment }\end{array}$ & $\begin{array}{c}\text { Hughes } \\
\text { \& Huth-Bocks }\end{array}$ & 2007 & $\begin{array}{c}\text { Examinar variações no estresse parental de } \\
\text { mulheres expostas à violência por parceiro } \\
\text { íntimo e relações entre funcionamento } \\
\text { materno e das crianças }\end{array}$ \\
\hline
\end{tabular}

Artigos excluídos após a classificação $(n=8)$

Motivo:

- Apresentavam, como participantes, além de crianças de zero a 12 anos, adolescentes, sem diferenciar resultados por idades. 
A7 - Domestic violence and longitudinal associations with children's physiological regulation abilities

A8 - Repercussão da exposição à violência por parceiro íntimo no comportamento dos filhos

\section{A9- Physically abused women's} experiences of sexual victimization and their children's disruptive behavior problems

A10 - Interparental violence, maternal emotional unavailability and children's cortisol functioning in family contexts

A11- Exposure to maternal and paternal perpetrated intimate partner violence, emotion regulation, and child outcomes

A12- Child involvement in interparental conflict and child adjustment problems: A longitudinal study of violent families

A13- PTSD symptoms in young children exposed to intimate partner violence

A14- Longitudinal impact of toddlers' exposure to domestic violence

$$
\begin{gathered}
\text { Rigterink, Katz, } \\
\text { \& Hessler }
\end{gathered}
$$

Durand, Schraiber, França, \& Barros
Spiller, Jouriles, McDonald, \& Skopp

Sturge-Apple, Davies, Cicchetti, \& Manning

Harding, Morelen, Thomassin, Bradbury, \& Shaffer

Jouriles, Rosenfield, McDonald, \& Mueller

Levendosky, Bogat, \& Martinez-Torteya

Schnurr

\& Lohman

2012
Examinar a relação entre exposição à violência doméstica e habilidades infantis para regular sua excitação fisiológica ao longo do tempo

2011 Analisar a associação entre a exposição à violência por parceiro íntimo contra $\mathrm{a}$ mulher com desajustes comportamentais e problemas escolares entre os filhos

Examinar se a violência física e sexual experimentada pela mulher através de seu parceiro está associada a problemas de comportamento disruptivo nos filhos

Examinar as especificidades da violência conjugal, indisponibilidade emocional materna e funcionamento fisiológico da criança nos relacionamentos interparentais e entre pais-filhos.

2013 Relação entre violência perpetrada por parceiro íntimo e sintomatologia de internalização e externalização em crianças.

Examinar se o envolvimento das crianças em conflitos interparentais prediz problemas de comportamentos de internalização e externalização nas crianças.

2013 Verificar associações entre violência por parceiro íntimo e sintomas de TEPT em crianças e suas mães.

Verificar como a exposição à violência doméstica na infância inicial pode aumentar a exposição a problemas na infância média. constituiu-se pelas mães ou cuidadoras (por ex.: avós, etc) e seus filhos, que responderam sobre a ocorrência ou não de violência conjugal. Já em três estudos (A2, A4, A7) os participantes foram o casal (pai ou padrasto, e mãe ou madrasta), e seus filhos. Por fim, um estudo (A8) teve como participantes somente as mães, vítimas de violência conjugal, que foram solicitadas a relatar sobre os comportamentos de seus filhos, sem que esses também se configurassem como participantes do estudo.

Ressalta-se que a maioria dos estudos (A1, A2, A4, A5, A7, A8, A9, A10, A11, A13, A14) contou com amostras selecionadas em diferentes ambientes comunitários, tais como escolas, hospitais, clínicas pediátricas, anúncios em jor- nais, e em instituições assistenciais que atendem pessoas que sofrem violência. Alguns estudos (A3, A6, A12) utilizaram amostras específicas relacionadas à violência conjugal, recrutadas em instituições de proteção a vítimas de violência.

\section{Características Metodológicas}

Nesta categoria são descritas, de forma breve, as características metodológicas dos estudos. Dos 14 artigos, apenas um (A8) é um estudo nacional, sendo todos os outros internacionais. Em relação ao delineamento, verificou-se que todos os estudos possuíam método quantitativo. Sobre a coleta de informações, em sua maioria, os estudos coletaram dados somente em um momento específico tendo sido estudos de corte transver- 
sal (A1, A2, A3, A4, A5, A6, A8, A9, A10 e A11) e apenas quatro (A7, A12, A13, A14) apresentaram delineamento longitudinal, coletando informações em diferentes momentos.

A coleta de informações deu-se, em sua maioria, através de instrumentos ou testes padronizados, mas também por questionários, entrevistas, observações, e procedimentos para avaliação de aspectos do funcionamento fisiológico da criança. $\mathrm{O}$ instrumento mais utilizado, em nove dos 14 estudos, foi o Child Behavior Checklist - CBCL, respondido pela mãe, para acessar os problemas de comportamento internalizantes e externalizantes nos filhos. Além deste, a observação da interação familiar (mãe, pai e filhos), bem como a observação da interação da criança com pares foi utilizada em um estudo (A2). Em outro (A1), além do CBCL, também foi utilizado o Attachment $Q$-set, respondido pelas mães, para acessar o padrão de apego estabelecido entre mãe-criança, bem como a observação do comportamento infantil pelo Eyberg task. Em outros três estudos (A3, A6, A11) também foi utilizado o Child Depression Inventory - CDI e o Revised Child Manifest Anxiety Scale - RCMAS (A3), ambos respondidos pelas crianças.

Cabe ressaltar ainda que um dos estudos (A9) utilizou a entrevista estruturada segundo o DSM-IV para acessar os comportamentos disruptivos infantis. Já outro (A8) utilizou-se de questionário padronizado para a pesquisa em questão. Em ambos os casos, os instrumentos foram aplicados somente às mães. Por fim, dois estudos avaliaram aspectos fisiológicos infantis (A7, A10), sendo que o primeiro, de Rigterink et al. (2010) utilizou eletrodos para medir a variação no espectro dos batimentos cardíacos $\mathrm{e}$ respiratórios de crianças expostas a violência conjugal, em uma atividade de laboratório. Foi considerada a linha de base tônus vagal, relacionada ao funcionamento do sistema nervoso central responsável pelo controle emocional, para acessar a capacidade de regulação emocional infantil. Já o estudo (A10) de Sturge-Apple et al. (2012) utilizou a coleta da saliva da criança para medir os níveis de cortisol no organismo, que se relaciona à vivência de situações estressantes. Além disso, também realizou a observação da interação mãe-criança pela técnica da Situação Estranha de Ainsworth, bem como uma observação da interação mãe-criança em jogo livre. Pode-se perceber que as características emocionais investigadas estão relacionadas a aspectos do desenvolvimento emocional infantil. No entanto, isso não fica explicitado nos artigos devido, em certa medida, às dificuldades em definir o que é e ao que se refere o desenvolvimento emocional.

A frequência, a gravidade e a exposição à violência conjugal foram acessadas por diferentes instrumentos nos estudos. A maior parte dos artigos utilizou o instrumento Conflict Tactics Scale - CTS que foi respondido por ambos os sujeitos do casal (A2, A4, A7), ou apenas pela mulher (A6, A9, A10, A11, A12, A14). Em outros três estudos (A1, A5 e A8), somente a mulher respondeu a instrumentos sobre a violência conjugal, sendo o Severity of Violence Against Women Scales - SVAWS (A1, A13), o Index Spousure Abuse - ISA-30 (A5), e um questionário padronizado para o estudo em questão (A8). Apenas em um estudo (A3) os respondentes foram os filhos expostos à violência conjugal, sendo acessados pelo The Children's Perceptions of Interparent Conflict Scale - CPIC.

Com relação ao subtipo de violência, a maior parte dos estudos investigou somente a agressão física (A2, A4, A7, A10, A12) como indicador de violência conjugal. Neste intuito, esses estudos consideraram para análise apenas a subescala de agressão física do Conflict Tactics Scale-CTS. Outro estudo (A11) utilizou as subescalas do CTS: violência física, ferimentos causados por agressão, coerção sexual e negociação. Já no estudo (A14) foram realizadas modificações na versão original do CTS, inserindo mais tipos de violência física e psicológica nas subescalas. Outros estudos destacaram os subtipos de violência física e sexual (A9); atos e ameaças de violência física e abuso sexual (A1); física e não física (A5); física, sexual e psicológica (A6, A8); e sem especificação dos subtipos de violência investigados (A3).

Em nove estudos (A1, A3, A5, A6, A8, A9, A12, A13, A14) foi dada atenção à violência sofrida pela mulher e perpetrada pelo companheiro, sem considerar a possibilidade de este 
também ser vítima. Ressalta-se que nos estudos que utilizaram o Conflict Tactics Scale - CTS (A6, A9), o instrumento por si já prevê o relato sobre violência cometida pelo entrevistado contra o cônjuge, assim como a violência sofrida pelo entrevistado. Mesmo assim, a definição do instrumento contida nos estudos mencionados, destacou que as mulheres respondiam sobre a violência cometida contra elas (e não por elas). Já outros estudos (A2, A4, A7, A10, A11) consideraram a agressão entre o casal e ressaltaram tanto a mulher quanto o homem como possíveis vítimas e autores da violência. Por exemplo, estudo (A2) de Katz e Low (2004) utilizou o Conflict Tactisc Scale - CTS para acessar a violência conjugal, sendo que o marido e a mulher responderam sobre os atos violentos cometidos por eles contra o(a) companheiro(a) nos últimos 12 meses.

Sobre a forma de exame dos dados dos estudos selecionados, em todos os artigos foram realizadas análises estatísticas, por meio de análises descritivas, caracterizando o perfil da amostra (idade, sexo, classe socioeconômica) e análises inferenciais, buscando observar os preditores ou as consequências da violência na mulher e nos filhos. Também foram utilizadas análises de modelagem de equações estruturais com vistas a propor modelos explicativos para a violência conjugal e suas consequências.

\section{Características EmocionaisInfantis}

Nesta categoria foram descritas, a partir dos objetivos dos estudos, bem como dos aspectos teóricos e metodológicos, as características emocionais infantis que podem ser afetadas pela exposição direta e/ou indireta à violência conjugal. Constatou-se que a maior parte dos estudos (A1, A2, A3, A4, A5, A6, A11, A12, A14) considerou, no seu foco de pesquisa, as características emocionais infantis relacionadas principalmente aos problemas de comportamento internalizantes e externalizantes que poderiam ser agravados ou gerados pela exposição à violência conjugal. Pode-se ressaltar o estudo (A3) de Skopp et al. (2005), que examinou se irmãos expostos à violência conjugal diferiam na sua percepção do conflito interparental e se tal percepção estava associada a diferenças no ajustamento emocional das crianças, considerando problemas de comportamento internalizantes e externalizantes. Além dos problemas de comportamento destacados, dois estudos (A1, A5) também investigaram as repercussões da violência conjugal no apego mãe-criança (A1) e na interação mãe-criança (A5).

Já outro estudo (A8) ressaltou a associação entre violência conjugal contra a mãe com comportamentos internalizantes e externalizantes (agressividade contra a mãe ou pares, urinar na cama, chupar o dedo, ter pesadelos frequentes, retraimento e fuga de casa) além de problemas escolares (abandono/interrupção ou repetência) nos filhos expostos a essa violência. Outro estudo (A9) investigou a presença de comportamento disruptivo em crianças expostas a violência conjugal, conforme os critérios do DSM-IV para diagnóstico de transtorno de conduta ou transtorno desafiador opositivo. Por fim, dois estudos (A7, A10) investigaram as reações fisiológicas infantis relacionadas à exposição à situação estressante de violência conjugal e suas repercussões para a capacidade da criança de regular suas emoções. Estudo (A7) de Rigterink et al. (2010) examinou a relação entre exposição à violência conjugal e habilidades infantis para regular sua excitação fisiológica ao longo do tempo. Os autores partiram do pressuposto de que crianças expostas à situação estressante de violência conjugal tenderiam a ter sua capacidade de regular emoções afetada, como dificuldades para se acalmarem sozinhas e maior necessidade de um suporte externo para a regulação emocional. Na mesma direção, o estudo (A10) de Sturge-Apple et al. (2012) examinou as especificidades da violência conjugal, da indisponibilidade emocional materna e do funcionamento fisiológico (resposta ao estresse) da criança nos relacionamentos interparentais e entre pais-filhos. Já o estudo (A13) de Levendosky et al. (2013) considerou os sintomas de Transtorno de Estresse Pós Traumático (TEPT), e sua relação com os mesmos sintomas apresentados pelas mães que sofriam violência pelo parceiro. 


\section{Repercussões da Exposição \\ à Violência Conjugal nas \\ Características Emocionais Infantis}

Nesta categoria foram investigadas as repercussões da exposição à violência conjugal nas características emocionais dos filhos com idades entre zero e 12 anos, a partir dos aspectos metodológicos, dos resultados e das discussões dos artigos. Todos os estudos utilizaram método quantitativo, sendo que através das análises descritivas e inferenciais foram acessados efeitos significativos ou moderados da exposição à violência conjugal no desenvolvimento dos filhos. maior parte dos estudos destacou a exposição da criança ao evento de violência entre os pais (testemunhar a violência); outros estudos, além disso, destacaram a exposição sofrida pelos agravos psicológicos causados no progenitor vítima da violência e sentida pela criança na interação com esse. Para fins de apresentação serão ressaltados, primeiramente, os resultados dos estudos compostos por amostras de mulheres que, em função da agressão, viviam em instituições de proteção, e em um segundo momento, daqueles que utilizaram amostras recrutadas em outros locais, como escolas, hospitais, clínicas pediátricas, anúncios em jornais, e em outras instituições assistenciais que atendem pessoas que sofrem violência.

Referente aos três estudos (A3, A6, A12) que utilizaram como amostra mulheres e filhos que viviam em instituições de proteção a vítimas de violência, pode-se ressaltar que foram encontrados efeitos significativos entre exposição à violência conjugal e características emocionais infantis investigadas. Por exemplo, o estudo (A3) de Skopp et al. (2005) investigou a percepção de irmãos sobre a violência conjugal ao qual eram expostos, e o quanto isso se relacionava a diferenças no ajustamento emocional entre eles. Para isso, 112 pares de irmãos (com médias de idade de dez e 12 anos) e suas mães fizeram parte do estudo. Os resultados revelaram que $21 \%$ das crianças apresentavam sintomatologia depressiva moderada a grave, $24 \%$ problemas de ansiedade moderada a grave, $38 \%$ problemas de comportamento externalizante, e $40 \%$ problemas de comportamento internalizante. Aqueles irmãos que relataram níveis mais elevados de sentimentos de ameaça e culpa em relação à violência conjugal tenderam a experienciar maiores problemas de ajustamento emocional, de modo que a percepção da gravidade da violência, e o se sentir ou não responsável por ela, pareceram influenciar no desenvolvimento infantil.

Já o estudo (A6) de Hughes e Huth-Bocks (2007) teve por objetivo examinar se existiam variações significativas no estresse parental entre mulheres expostas à violência por parceiro íntimo e se esses padrões relacionavam-se com diferenças em outras áreas do funcionamento materno e da criança. Participaram 172 díades mãe-criança e foi considerada, como foco do estudo, a exposição indireta da criança à violência conjugal, a partir dos danos psíquicos maternos e na relação mãe-filho. Os dados apontaram que as mães apresentavam altos níveis de estresse que repercutiam no exercício dos seus papéis parentais e nas interações com a criança. As mães experienciavam sentimentos de incompetência quanto à capacidade de ser mãe e consideravam as interações com os filhos como desagradáveis e estressantes. As mães que obtiveram altos níveis de estresse foram as que perceberam maiores problemas de comportamento e emocionais nos filhos, sendo que $40 \%$ das crianças foram classificadas como apresentando problemas de internalização (timidez, retraimento) e $52 \%$ problemas de externalização (maior agressividade).

Com relação aos estudos que utilizaram amostras recrutadas em diferentes contextos comunitários (escolas, hospitais, abrigos, anúncios em jornal, subamostras de outros estudos maiores sem especificação, etc; A1, A2, A4, A5, A7, A8, A9, A10, A11, A13, A14), os efeitos da exposição à violência conjugal nas características emocionais infantis investigadas foram encontrados. No entanto, a severidade da violência tendeu a ser menos grave e o efeito da violência no desenvolvimento infantil foi mais moderado que a dos estudos em que mães e crianças estavam acolhidas em instituições de proteção às vítimas de violência. Por exemplo, estudo (A2) de Katz e Low (2004) investigou a relação entre violência doméstica, coparentalidade e processos familiares, e sua relação com o ajustamento psicológico infantil, em 130 famílias com filhos em idade 
pré-escolar. Nesse estudo, a violência conjugal foi relacionada a outras dinâmicas de funcionamento dentro da família, como a coparentalidade, definida pelos autores como as maneiras pelas quais os parceiros apoiam-se mutuamente no seu papel conjunto como líderes da família. Os dados indicaram que casais violentos tenderam a exercer uma coparentalidade mais conflituosa, sendo que ao invés de esses casais apoiarem uns aos outros nos seus papéis parentais, acabavam mais suscetíveis a envolver o outro na relação de uma forma mais negativa e crítica. Por sua vez, a violência conjugal esteve relacionada com níveis mais elevados de não cumplicidade da criança ao interagir com seus pares e com dificuldades em regular suas emoções para interagir socialmente. Da mesma forma, a coparentalidade mais hostil mediou a relação entre violência conjugal e ansiedade/depressão nas crianças, o que sugere que em lares maritalmente violentos, a coparentalidade tende a ser mais crítica e hostil, colocando os filhos em risco de desenvolver maior sintomatologia depressiva e ansiosa.

No estudo longitudinal (A10) de Rigterink et al. (2010) foi examinada a relação entre exposição à violência conjugal e habilidades infantis para regular a excitação fisiológica ao longo do tempo. Para isso, participaram da pesquisa 68 famílias, nas quais as crianças eram submetidas a um exame por eletrodos para medir a frequência cardíaca e respiratória frente a uma atividade em laboratório (escutar histórias por fone de ouvido). Essa atividade buscou avaliar a regulação emocional infantil frente a uma situação estressante, medida pela linha de base tônus vagal, indicador do funcionamento do sistema nervoso parassimpático. O estudo iniciou com 130 famílias, sendo que em metade delas as crianças foram classificadas como apresentando problemas de conduta, avaliados pelo Eyberg Child Behavior Inventory, e a outra metade classificada como sem problemas de conduta, formando dois grupos distintos de análise. Ainda havia subdivisão dentro dos grupos, classificando aquelas crianças expostas e não expostas à violência conjugal. Essas famílias foram acessadas em dois momentos, sendo o primeiro quando as crianças estavam com idades entre quatro e seis anos, e o segundo momento quatro anos após o primeiro encontro, havendo perda de muitos participantes e finalizando o estudo com 68 famílias. Os dados revelaram que a gravidade da violência doméstica testemunhada pelas crianças foi baixa, já que os atos violentos predominantes foram o empurrar, o agarrar ou o jogar algo no parceiro. Tanto homens quanto mulheres relataram os atos violentos perpetrados por si contra o cônjuge, o que coloca ambos como possíveis vítimas e autores da violência. Foram encontradas evidências de diferenças na trajetória do tônus vagal ao longo do tempo, sendo que as crianças expostas à violência conjugal apresentaram aumentos menores na linha de base tônus vagal do que as não expostas, indicando que a exposição à violência conjugal afeta negativamente o desenvolvimento de capacidades reguladoras emocionais internas. Isso sugere que a violência conjugal pode ter consequências negativas não só a curto prazo, mas também prejudicar o funcionamento emocional ao longo do tempo.

\section{Discussão}

A maioria dos artigos selecionados para este estudo não explicitou uma clara definição de violência conjugal. No entanto, os autores investigaram diversas formas de violência (física, sexual e psicológica) a que foram expostos mulheres, crianças e/ou casais dos estudos. Cabe ressaltar que a maior parte das formas de violência descritas foram aquelas que incluem agressões físicas, embora três estudos tenham incluído a violência psicológica. Destaca-se a dificuldade de identificação da violência psicológica, que inclui, entre outras formas de violência, a agressão verbal, chantagens, ameaças e humilhações, ao contrário da violência física que resulta em marcas corporais (Ministério da Saúde, 2001). Esse fato pode fazer com que nem sempre as vítimas percebam o ato como uma forma de violência (Brum et al., 2013), embora a violência psicológica geralmente ocorra concomitantemente com outros tipos de violência estando, portanto, quase sempre presente (Ministério da Saúde, 2001).

Nos estudos investigados, a exposição à violência conjugal foi considerada a partir das 
agressões sofridas pela mulher em relação ao parceiro, embora alguns artigos tenham mencionado a violência conjugal ocorrida entre os parceiros. Considerou-se, dessa forma, a violência que é sofrida pelo homem, e não somente pela mulher. Em uma perspectiva sistêmica, a violência é considerada como um continuum em que a exposição de um membro familiar à violência acaba interferindo de forma direta ou indireta, em todas as relações (Bhona et al., 2011; Colossi \& Falcke, 2013; Preto \& Moreira, 2012), embora apenas um dos estudos analisados embase explicitamente sua discussão nessa perspectiva (A12). Por outro lado, mesmo que o casal não agrida seus filhos, o fato da criança testemunhar a violência tende a repercutir negativamente no desenvolvimento infantil, de acordo com estudos que buscam uma visão mais ampliada do fenômeno (Hardaway et al., 2012; Mrug \& Windle 2010).

Com isso, pode-se destacar que a violência tende a influenciar todos os membros da família, seja de forma direta (ser a pessoa agredida) quanto indireta (testemunhar a agressão), podendo causar consequência a curto, médio e logo prazo. A fim de compreender as consequências para o desenvolvimento de crianças de zero a 12 anos, este estudo considerou os aspectos metodológicos, resultados e discussões dos artigos que remetiam às repercussões da exposição à violência conjugal nas características emocionais infantis. A ausência de uma teoria de base e de definições mais claras sobre o desenvolvimento emocional colaborou, em certa medida, para as dificuldades em se investigar esse conceito nos estudos empíricos selecionados. Dessa forma, os estudos ficaram centrados em aspectos mais objetivos de comportamento, de presença ou ausência de sintomas que indicavam problemas de comportamentos internalizantes e externalizantes, conflitos nas relações mãe-criança, dentre outros. Além disso, apenas um estudo (A12) contemplou os aspectos transgeracionais da violência, de forma a analisar as histórias de violência do casal ou como essa violência conjugal atual poderá repercutir no comportamento violento dos filhos em suas relações posteriores - relação já estudada por alguns autores sendo um aspecto importante ao considerarem-se as causas e manutenção da violência conjugal (Colossi \& Falcke, 2013; Marasca et al., 2013; Santos \& Moré, 2011).

No que tange às repercussões da exposição à violência, verificou-se que essa tende a apresentar repercussões no desenvolvimento, seja de forma direta, em relação à criança vítima, seja de forma indireta, a partir dos danos psicológicos provocados à mãe agredida e que afetam a relação mãe-criança, por exemplo. Teorias clássicas consideram que as relações estabelecidas com os cuidadores primários, em especial a mãe, reverberam ao longo do ciclo vital (Bowlby, 1989; Mahler et al., 1975/1977; Winnicott, 1945/2000). Dessa forma, relações afetivas mais empobrecidas entre mãe-criança, prejudicadas pelos efeitos da violência, tendem a afetar o desenvolvimento e saúde mental dos filhos. Nesse sentido, todos os artigos investigados demonstraram que a violência conjugal, mesmo isolada, repercute nos outros membros da família, em especial nos filhos, mesmo que não deixem claro se as crianças, além de testemunharem a violência conjugal, também eram vítimas da violência. Tendo em vista que em ambientes violentos, essa tende a ser estendida para outros membros da família e não se centrar apenas no casal, é importante que a violência conjugal seja compreendida de forma mais ampla, já que o impacto dessas situações ultrapassa as relações agressor/agredido (Baldry, 2007; Bhona et al., 2011; Hunter \& Graham-Bermann, 2013; Preto \& Moreira, 2012).

A predominância de estudos quantitativos permitiu observar se a exposição à violência conjugal exerce impacto significativo no desenvolvimento infantil, principalmente relacionado a características emocionais. No entanto, as metodologias utilizadas nos estudos selecionados não permitiram aprofundar aspectos subjetivos que perpassam essa violência e que possibilitariam uma maior compreensão da dinâmica familiar em contexto de violência conjugal. Para futuros estudos, sugere-se considerar a abordagem qualitativa ou mista para uma maior compreensão do fenômeno.

Outro fator observado foi a predominância de estudos transversais que permitem acessar o dado naquele momento e contexto de desenvol- 
vimento infantil. Estudos longitudinais permitiriam entender, de maneira mais aprofundada, o efeito da exposição à violência conjugal ao longo do desenvolvimento da criança, considerando as especificidades que são inerentes a cada idade.

Ressalta-se que essa revisão de literatura aponta para a escassez de estudos que consideram as vítimas indiretas da violência, ou seja, aquelas que não são agredidas, mas testemunham a agressão entre os pais e, por esse motivo, também sofrem suas consequências. Na seleção final, apenas um estudo nacional e 13 internacionais foram analisados. Esse dado demonstra a necessidade de novos estudos sobre as repercussões da exposição à violência conjugal no desenvolvimento dos filhos, já que há uma alta prevalência desse fenômeno nas famílias brasileiras e no mundo (Ministério da Saúde, 2001; Waiselfisz, 2012). No entanto, ressalta-se que os descritores e as bases de dados utilizadas na busca podem ter restringido o número de artigos encontrados sobre o tema. Assim, sugere-se que em novos estudos possam ser utilizadas outras bases de dados, como Web of Science e Scopus, por exemplo.

Ainda, os estudos demonstraram, a partir de seus resultados, a necessidade e a importância de acompanhar todos os envolvidos em um lar violento, e não somente as vítimas diretas (aquelas que são agredidas). Em uma compreensão sistêmica, toda a família sofre as consequências da violência conjugal, sendo que vítima e agressor podem ocupar ambos os papéis. Da mesma forma, torna-se importante investigar as consequências da exposição à violência conjugal em outras áreas do desenvolvimento não mencionadas nos artigos em questão, como as relações mais conflitivas entre os próprios irmãos, as dificuldades em estabelecer amizades, os problemas de aprendizagem em decorrência de fatores emocionais, a transferência da agressividade vivenciada em casa para o ambiente escolar, dentre outros aspectos.

\section{Considerações Finais}

A partir da revisão sistemática realizada, percebeu-se, em termos metodológicos, a ne- cessidade de estudos que permitam o aprofundamento e compreensão da violência conjugal, definindo de forma explícita o que os autores entendem por violência na relação do casal e como esta influencia o desenvolvimento dos filhos. No que diz respeito às características emocionais, também foi observada uma falta de teorização acerca desse conceito e uma dificuldade de conceituá-lo. Os autores nem sempre deixaram claro o que entendem por desenvolvimento emocional, dificultando a análise dos dados e a compreensão das repercussões da exposição à violência conjugal.

De maneira geral, os estudos destacaram as repercussões da exposição à violência conjugal nas características emocionais dos filhos, demonstrando que a violência, além de repercutir de maneira negativa no casal - e, principalmente na mulher (foco da maior parte dos artigos) - também implica em consequências importantes para outros membros da família, como as crianças.

Dessa forma, as intervenções em famílias expostas à violência devem abarcar a compreensão da dinâmica familiar, de forma a assegurar primeiramente, a segurança do(s) filho(s) e da vítima direta da violência conjugal - geralmente a mulher. Os serviços de saúde e proteção têm como dever garantir o acolhimento e atendimento das vítimas da violência, com base em acompanhamento médico, psicológico e social, assim como a proteção da pessoa agredida. Além disso, o atendimento aos agressores também deve ocorrer, sem que isso signifique a não responsabilização pelo ato violento (Ministério da Saúde, 2010). Em uma visão sistêmica, a violência conjugal é considerada um problema da dinâmica relacional do casal sendo, portanto, necessárias intervenções junto à díade, buscando a resolução de conflitos e relações familiares mais saudáveis.

Trabalhar na prevenção da violência também é uma prática importante, visto que no Brasil, há histórica e culturalmente uma banalização da violência, principalmente em relação às mulheres e as crianças. A disseminação de conhecimentos sobre as repercussões da violência no desenvolvimento das crianças, adolescentes e da família, é essencial, sobretudo porque ainda há uma naturalização de tais práticas violentas. 
Ampliar o olhar sobre a violência conjugal é "tornar visível o invisível", em uma tentativa de romper e desnaturalizar a história de violência no contexto familiar.

\section{Referências}

Abranches, C., \& Assis, S. (2011). A (in)visibilidade da violência psicológica na infância e na adolescência no contexto familiar. Cadernos de Saúde Pública, 27(5), 843-854. doi:S0102$-311 \mathrm{X} 2011000500003$

Almeida, A., Miranda, O., \& Lourenço, L. (2013). Violência doméstica/intrafamiliar contra crianças e adolescentes: Uma revisão bibliométrica. Gerais: Revista Interinstitucional de Psicologia, 6(2), 298-311. Recuperado em http://pepsic. bvsalud.org/pdf/gerais/v6n2/v6n2a11.pdf

Araújo, M. F. (2002). Violência e abuso sexual na família. Psicologia em Estudo, 7(2), 3-11. Recuperado em http://www.scielo.br/pdf/pe/ $\mathrm{v} 7 \mathrm{n} 2 / \mathrm{v} 7 \mathrm{n} 2 \mathrm{a} 02 . p d f$

Baldry, A. C. (2007). "It does affect me": Disruptive behaviors in preadolescents directly and indirectly abused at home. European Psychologis, 12(1), 29-35. doi:10.1027/1016-9040.12.1.29

Benetti, S. P. C. (2006). Violência conjugal: Impacto no desenvolvimento psicológico da criança e do adolescente. Psicologia: Reflexão e Crítica, 19(2), 261-268. doi:10.1590/S010279722006000200012

Benetti, S. P. C., Pizetta, A., Schwartz, C. B., Hass, R. A., \& Melo, V. L. (2010). Problemas de saúde mental na adolescência: Características familiares, eventos traumáticos e violência. Psico-USF, 15(3), 321-332.

Bhona, F., Lourenço, L., \& Brum, C. (2011). Violência doméstica: Um estudo bibliométrico. $A r$ quivos Brasileiros de Psicologia, 63(1), 87-100. Recuperado em http://pepsic.bvsalud.org/pdf/ $\operatorname{arbp} / \mathrm{v} 63 \mathrm{n} 1 / \mathrm{v} 63 \mathrm{n} 1 \mathrm{a} 10 . \mathrm{pdf}$

Braga, L. L., \& Dell'Aglio, D. D. (2012). Exposição à violência em adolescentes de diferentes contextos: Família e instituição. Estudos de Psicologia (Natal), 17(3), 413-320. doi:10.1590/S1413294X2012000300009. Recuperado em http:// www.scielo.br/pdf/epsic/v17n3/09.pdf

Brancalhone, P., Fogo, J. C., \& Williams, L. C. (2004). Crianças expostas à violência conjugal:
Avaliação do desempenho acadêmico. Psicologia: Teoria e Pesquisa, 20(2), 113-117. Recuperado em http://www.scielo.br/pdf/ptp/v20n2/ a03v20n2

Brum, C., Lourenço, L., Gebara, C., \& Ronzani, T. (2013). Violência doméstica e crenças: Intervenção com profissionais da atenção primária em saúde. Psicologia em Pesquisa, 7(2), 242-250. doi:10.5327/Z1982-1247201300020012

Bowlby, J. (1989). Uma base segura: Aplicações clínicas da teoria do apego. Porto Alegre, RS: Artes Médicas.

Colossi, P. M., \& Falcke, D. (2013). Gritos do silêncio: A violência psicológica no casal. Psico, 44(3), 310-318. Recuperado em http://revistaseletronicas.pucrs.br/fabio/ojs/index.php/revistapsico/article/view/11032

Cortez, M., Cruz, G., \& Souza, L. (2013). Violência conjugal: Desafios e propostas para a aplicação da Lei Maria da Penha. Psico, 44(3), 499507. Recuperado em http://revistaseletronicas. pucrs.br/ojs/index.php/revistapsico/article/ view/11542

Durand, J. G., Schraiber, L. B., França, I., Jr., \& Barros, C. (2011). Repercussões da exposição à violência por parceiro íntimo no comportamento dos filhos. Revista de Saúde Pública, 45(2), 355364. doi:10.1590/S0034-89102011005000004. Recuperado em http://www.scielo.br/pdf/rsp/ v45n2/1940.pdf

Hanson, R., Self-Brown, S., Borntrager, C., Kilpatrick, D., Saunders, B., Resnick, H., \& Amstadter, A. (2008). Relations among gender, violence exposure and mental health: The national survey of adolescents. American Journal of Orthopsychiatry, 3, 313-321. doi:10.1037/a0014056

Haynie, D., Petts, R., Maimon, D., \& Piquero, A. (2009). Exposure to violence in adolescence and precocious role exits. Journal Youth Adolescence, 38, 269-286. doi:10.1007/s10964-0089343-2

Hardaway, C., McLoyd, V., \& Wood, D. (2012). Exposure to violence and socioemotional adjustment in low-income youth: An examination of protective factors. American Journal Community Psychology, 49, 112-126. doi:10.1007/ s10464-011-9440-3

Harding, H., Morelen, D., Thomassin, K., Bradbury, L., \& Shaffer, A. (2013). Exposure to maternal and paternal perpetrated intimate partner 
violence, emotion regulation, and child outcomes. Journal Family Violence, 28, 63-72. doi:10.1007/s10896-012-9487-4

Hart, S., Hodgkinson, S., Belcher, H., Hyman, C., \& Cooley-Strickland, M. (2012). Somatic symptoms, peer and school stress, and family and community violence exposure among urban elementary school children. Journal Behavioral Medicine, 15, 1-8.

Ho, M. Y., \& Cheung, F. M. (2010). The differential effects of forms and settings of exposure to violence on adolescents' adjustment. Journal of Interpersonal Violence, 25(7), 1309-1337. doi:10.1177/0886260509340548

Hughes, H., \& Huth-Bocks, A. (2007). Variations in parenting stress in African-American battered women: Implications for children's adjustment. European Psychologist, 12(1), 6271. doi:10.1027/1016-9040.12.1.62

Hunter, E., \& Graham-Bermann, S. (2013). Intimate partner violence and child adjustment: Moderation by father contact? Journal Family Violence, 28, 435-444. doi:10.1007/s10896-013-9517-x

Jouriles, E., Rosenfield, D., McDonald, R., \& Mueller, V. (2013). Child involvement in interparental conflict and child adjustment problems: A longitudinal study of violent families. Journal of Abnormal Child Psychology, 42, 1-12. doi:10.1007/s10802-013-9821-1.

Katz, L. F., \& Low, S. M. (2004). Marital violence, co-parenting, and family-level processes in relation to children's adjustment. Journal of Family Psychology, 18(2), 372-382. doi:10.1037/08933200.18.2.372

Katz, L. F., \& Windecker-Nelson, B. (2006). Domestic violence, emotion coaching, and child adjustment. Journal of Family Psychology, 20(1), 56-67. doi:10.1037/0893-3200.20.1.56

Lei $\mathrm{N}^{\circ} 11.340$, de 07 de agosto de 2006. (2006, 08 ago.). Cria mecanismos para coibir a violência doméstica e familiar contra a mulher. Diário Oficial da União. Recuperado em http://www. planalto.gov.br/ccivil_03/_ato2004-2006/2006/ lei/111340.htm

Levendosky, A., Bogat, A., \& Martinez-Torteya, C. (2013). PTSD symptoms in young children exposed to intimate partner violence. Violence Against Women, 19(2), 187-201. doi: $10.1177 / 1077801213476458$
Levendosky, A., Huth-Bocks, A., Shapiro, D., \& Semel, M. (2003). The impact of domestic violence on the maternal-child relationship and preschool-age children's functioning. Journal of Family Psychology, 17(3), 275-287. doi:10.1037/0893-3200.17.3.275

Mahler, M., Pine, F., \& Bergman, A. (1977). O nascimento psicológico da criança. Rio de Janeiro, RJ: Zahar Editores. (Original publicado em 1975)

Marasca, A., Colossi, P., \& Falcke, D. (2013). Violência conjugal e família de origem: Uma revisão sistemática da literatura de 2006 a 2011. Temas em Psicologia, 21(1), 221-243. doi:10.9788/ TP2013.1-16

Margolin, G., Vickerman, K., Oliver, P., \& Gordis, E. B. (2010). Violence exposure in multiple interpersonal domains: Cumulative and differential effects. Journal of Adolescent Health, 47, 198205. doi:j.jadohealth.2010.01.020

Minayo, M. C. (2006). Contextualização do debate sobre violência contra crianças e adolescentes. In Ministério da Saúde (Ed.), Violência faz mal à saúde (pp. 13-16). Brasília, DF: Ministério da Saúde.

Ministério da Saúde. (2001). Violência intrafamiliar: Orientações para a prática em serviço. Cadernos de Atenção Básica, 8(131), 1-100. Recuperado em http://bvsms.saude.gov.br/bvs/publicacoes/ cd05_19.pdf

Ministério da Saúde. (2010). Linha de cuidado para atenção integral à saúde de crianças, adolescentes e suas famílias em situação de violências: Orientação para gestores e profissionais da saúde. Brasília, DF: Autor. Recuperado em http:// bvsms.saude.gov.br/bvs/publicacoes/linha_cuidado_criancas_familias_violencias.pdf

Miranda, M. P. M., Paula, C. S., \& Bordin, I. A. (2010). Violência conjugal física contra a mulher na vida: Prevalência e impacto imediato na saúde, trabalho e família. Revista Panamericana de Salud Publica, 27(4), 300-308. doi:10.1590/ S102049892010000400009

Mrug, S., \& Windle, M. (2010). Prospective effects of violence exposure across multiple contexts on early adolescents' internalizing and externalizing problems. Journal of Child Psychology and Psychiatry, 51(8), 953-961. doi:10.1111/j.14697610.2010.02222.x 
Oliveira, K., \& Gomes, R. (2011). Homens e violência conjugal: Uma análise de estudos brasileiros. Ciência \& Saúde Coletiva, 16(5), 2401-2413. doi:10.1590/S141381232011000500009

Owen, A., Kaslow, N., \& Thompson, M. (2006). The mediating role of parenting stress in the relation between intimate partner violence and child adjustment. Journal of Family Psychology, 20(3), 505-513. doi:10.1037/0893-3200.20.3.505

Preto, M., \& Moreira, P. (2012). Auto-regulação da aprendizagem em crianças e adolescentes filhos de vítimas de violência doméstica contra mulheres. Psicologia: Reflexão e Crítica, 25(4), 730737. doi:10.1590/S0102-79722012000400012. Recuperado em http://www.scielo.br/pdf/prc/ v25n4/12.pdf

Rigterink, T., Katz, L., \& Hessler, D. (2010). Domestic violence and longitudinal associations with children's physiological regulation abilities. Journal of Interpersonal Violence, 25(9), 16691683. doi:10.1177/0886260509354589

Santos, A., \& Moré, C. (2011). Impacto da violência no sistema familiar de mulheres vítimas de agressão. Psicologia: Ciência $e$ Profissão, 31(2), 220-235. doi:10.1590/S141498932011000200003

Santos, K., Santos, L., Lima L., Brito, L, Silva, Y., \& Gonçalves, H. (2014). A violência doméstica contra a mulher por companheiro e a Lei Maria da Penha. Cadernos de Graduação - Ciências Humanas e Sociais, 1(2), 79-86. Recuperado em https://periodicos.set.edu.br/index.php/cadernohumanas/article/view/1259/706

Schnurr, M., \& Lohman, B. (2013). Longitudinal impact of toddlers' exposure to domestic violence. Journal of Aggression, Maltreatment \& Trauma, 22, 1015-1031. doi:10.1080/10926771 .2013 .834019

Skopp, N., Manke, B., McDonald, R.., \& Jouriles, E. (2005). Siblings in domestically violent families: Experiences of interparental conflict and adjustment problems. Journal of Family Psychology, 19(2), 324-333. doi:10.1037/08933200.19.2.324

Spiller, L., Jouriles, E., McDonald, R., \& Skopp, N. (2012). Physically abused women's experiences of sexual victimization and their children's disruptive behavior problems. Psychology of Violence, 2(4), 401-410. doi:10.1037/a0028912
Sturge-Apple, M., Davies, P., Cicchetti, D., \& Manning, L. (2012). Interparental violence, maternal emotional unavailability and children's cortisol functioning in family contexts. Developmental Psychology, 48(1), 237-249. doi:10.1037/ a0025419

Vieira, E. M., Perdona, G. S. C., \& Santos, M. A. (2011). Fatores associados à violência física por parceiro íntimo em usuárias de serviços de saúde. Revista de Saúde Pública, 45(4), 730-737. doi:10.1590/S0034-89102011005000034

Waiselfisz, J. J. (2012). Mapa da violência 2012: Homicídio de mulheres no Brasil. Rio de Janeiro, RJ: Cebela.

World Health Organization. (2002). Version of the introduction to the world report on violence and health. Geneva, Switzerland: Author. Retrieved from http://www.who.int/violence_injury_prevention/violence/world_report/en/introduction. pdf

Williams, L. C. A., D’Affonseca, S. M., Correia, T. A., \& Albuquerque, P. P. (2011). Efeitos a longo prazo de vitimização na escola. Gerais: Revista Interinstitucional de Psicologia, 4(2), 187-199. Recuperado em http://www.laprev.ufscar.br/ documentos/arquivos/artigos/2011-williams-edaffonseca.pdf

Williams, L. C. A., \& Stelko-Pereira, A. C. (2008). A associação entre violência doméstica e violência escolar: Uma análise preliminar. Educação: Teoria e Prática, 18(30), 25-35. Recuperado em http://www.ppgees.ufscar.br/a-associacao-entre-violencia-domestica-e-violencia-e-scolar-uma-analise-preliminar

Winnicott, D. W. (2000). Desenvolvimento emocional primitivo. In D. W. Winnicott (Ed.), Da pediatria à psicanálise (pp. 218-232). Rio de Janeiro, RJ: Imago. (Original publicado em 1945) 